LBNL- 58259

\title{
The Limits of Quintessence
}

\author{
R. R. Caldwell \\ Department of Physics \& Astronomy, Dartmouth College, Hanover, NH 03755 \\ Eric V. Linder \\ Physics Division, Lawrence Berkeley Laboratory, Berkeley, CA 94720
}

\begin{abstract}
This work was supported in part by the Director, Office of Science, Office of High Energy Physics, of the U.S. Department of Energy under Contract No. DE-AC03-76SF00098.
\end{abstract}

DISCLAIMER

This document was prepared as an account of work sponsored by the United States Government. While this document is believed to contain correct information, neither the United States Government nor any agency thereof, nor The Regents of the University of California, nor any of their employees, makes any warranty, express or implied, or assumes any legal responsibility for the accuracy, completeness, or usefulness of any information, apparatus, product, or process disclosed, or represents that its use would not infringe privately owned rights. Reference herein to any specific commercial product, process, or service by its trade name, trademark, manufacturer, or otherwise, does not necessarily constitute or imply its endorsement, recommendation, or favoring by the United States Government or any agency thereof, or The Regents of the University of California. The views and opinions of authors expressed herein do not necessarily state or reflect those of the United States Government or any agency thereof or The Regents of the University of California. 


\title{
The Limits of Quintessence
}

\author{
R. R. Caldwell \\ Department of Physics \& Astronomy, Dartmouth College, Hanover, NH 03755 \\ Eric V. Linder \\ Physics Division, Lawrence Berkeley Laboratory, Berkeley, CA 94720
}

(Dated: February 2, 2008)

\begin{abstract}
We present evidence that the simplest particle-physics scalar-field models of dynamical dark energy can be separated into distinct behaviors based on the acceleration or deceleration of the field as it evolves down its potential towards a zero minimum. We show that these models occupy narrow regions in the phase-plane of $w$ and $w^{\prime}$, the dark energy equation-of-state and its time-derivative in units of the Hubble time. Restricting an energy scale of the dark energy microphysics limits how closely a scalar field can resemble a cosmological constant. These results, indicating a desired measurement resolution of order $\sigma\left(w^{\prime}\right) \approx(1+w)$, define firm targets for observational tests of the physics of dark energy.
\end{abstract}

Observations and experiments at the close of the 20th century have transformed our understanding of the physics of the Universe. A consistent picture has emerged indicating that nearly three-quarters of the cosmos is made of "dark energy" - some sort of gravitationally repulsive material responsible for the accelerated expansion of the Universe (for reviews see [1, 2, 3]). Proposals for the dark energy include Einstein's cosmological constant $(\Lambda)$, or a dynamical field such as quintessence. Here we show how scalar field dynamics separates into distinct behaviors which, through future cosmological measurements, can reveal the nature of the new physics accelerating our universe.

Einstein's cosmological constant $(\Lambda)$ is attributed to the quantum zero-point energy of the particle physics vacuum, with a constant energy density $\rho$, pressure $p$ and an equation-of-state $w \equiv p / \rho=-1$. In contrast, quintessence is a proposed time-varying, inhomogeneous field with a spatially-averaged equation-of-state $w>-1$ [4, 5, 6, 7, 8]. The simplest physical model consists of a scalar field, slowly rolling in a potential characterized by an extremely low mass. (This is similar to inflation, the period of accelerated expansion in the early universe, but at an energy scale many orders of magnitude lower.) Since a scalar field evolving in a very shallow potential may be indistinguishable from a $\Lambda$, the task of elucidating the physics of dark energy becomes difficult if observations continue to find that $w$ is close to -1 , e.g. 9, 10, 11]. In this letter, we examine the likely behavior of scalar fields and characterize them into two distinct classes, based on their evolution in the $w-w^{\prime}$ phase space. These results should help define targets for observational and experimental tests of the physics of dark energy.

Our approach is a new take on a familiar system, the scalar field. By emphasizing the dynamics, we discover restricted regions of the trajectories of canonical scalar field models in "position" and "velocity" — the value of the equation-of-state ratio $w$ and its time variation $w^{\prime}$. While there is a myriad of scalar field models mo- tivated by particle physics beyond the standard model, this treatment allows a broad, model-independent assessment of a quintessence scalar field slowly relaxing in a potential.

The physics is straightforward: the field $\phi$ will seek to roll towards the minimum of its potential $V$, according to the Klein-Gordon equation $\ddot{\phi}+3 H \dot{\phi}=-d V / d \phi$. The rate of evolution is driven by the slope of the potential and damped by the cosmic expansion through the Hubble

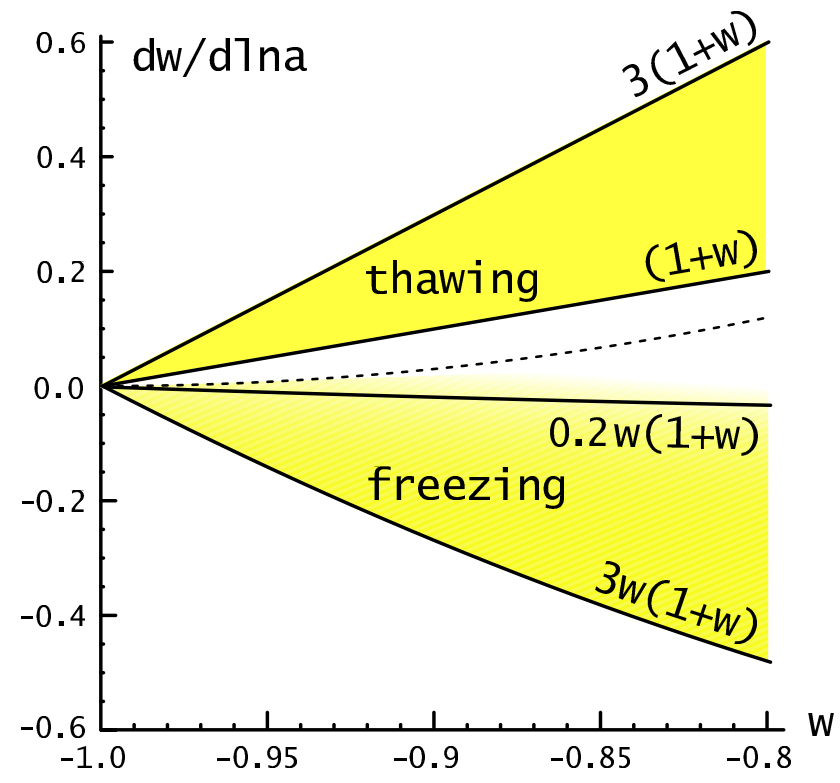

FIG. 1: The $w-w^{\prime}$ phase space occupied by thawing and freezing fields is indicated by the shaded regions. No strong constraints on this range of dark energy properties exist at present. The fading at the top of the freezing region indicates the approximate nature of this boundary. Freezing models start above this line, but pass below it by a red shift $z \sim 1$. The short-dashed line shows the boundary between field evolution accelerating and decelerating down the potential. Future cosmological observations will aim to discriminate between these two fundamental scenarios. 
parameter $H$. The average energy density and pressure are $\rho=\dot{\phi}^{2} / 2+V, p=\dot{\phi}^{2} / 2-V$ so that a field stuck in a local, non-zero minimum of the potential has $w=-1$. To distinguish from an effective cosmological constant, however, we will only consider cases in which the field is evolving towards a zero minimum.

In perhaps the simplest such scenario, the field has been frozen by Hubble damping at a value displaced from its minimum until recently, when it starts to roll down to the minimum. We call these "thawing" models. At early times the equation-of-state ratio is $w \approx-1$, but grows less negative with time as $w^{\prime} \equiv \dot{w} / H>0$. Since the Hubble damping limits the scalar field acceleration, $\ddot{\phi}<\dot{\phi} / t \approx(3 / 2) H \dot{\phi}$, then the equation of motion implies such models will lie at $w^{\prime}<3(1+w)$ in the phase plane. The scalar field dynamics suggest a lower bound, too, due to the fact that dark energy is not entirely dominant today, with a fractional energy density $\Omega_{d e} \lesssim 0.8$. A study of several classes of thawing models, such as a pseudo Nambu-Goldstone boson (PNGB) [6] or polynomial potentials, indicates the bound $w^{\prime}>(1+w)$. These simple bounds are valid for $(1+w) \ll 1$, and so $w \lesssim-0.8$ is a practical limit of applicability.

We have analyzed the following potentials for thawing behavior: Concave potentials with $V=M^{4-n} \phi^{n}$ are ubiquitous, and we have allowed for continuous values of the exponent $n>0$. The motivation for cases $n<2$ is not as straightforward, although $n=1$ has been considered 12]. Exponential potentials are typical for moduli or dilaton fields, e.g. 13, with $V=M^{4} \exp \left(-\beta \phi / M_{P}\right)$ where $M_{P} \approx 10^{19} \mathrm{GeV}$ is the Planck energy. To avoid scaling, which would not provide for the cosmic acceleration, we restrict $\beta<\sqrt{24 \pi}$ 14. PNGBs, like a dark energy axion, have $V=M^{4} \cos ^{2}(\phi / 2 f)$ where $f$ is a symmetry restoration energy scale. We have not included the case $f \ll M_{P}$ since the field rapidly evolves to $w \rightarrow 0$ unless the initial conditions are finely tuned to keep the field balanced upon the top of the potential maxima and maintain $1+w \ll 1$.

A second scenario consists of a field which was already rolling towards its potential minimum, prior to the onset of acceleration, but which slows down and creeps to a halt as it comes to dominate the universe. For these "freezing" models, initially $w>-1$ and $w^{\prime}<0$. These are essentially tracking models [15], but may be described more generally as vacuumless fields (in the sense that the minimum is attained as $\phi \rightarrow \infty$ ) or runaway potentials characterized by a potential with curvature that slows the field evolution as it rolls down towards the minimum. It follows [16] that there is some value of the field beyond which the evolution is critically damped by the cosmic expansion, whence the field is frozen (but, like a glacier [16], continues to move) and $w \rightarrow-1, w^{\prime} \rightarrow 0$. The deceleration of the field is limited by the steepness of the potential, roughly $\ddot{\phi}>d V / d \phi$, leading to the lower bound $w^{\prime}>3 w(1+w)$. Investigation of a variety of scalar field models leads to a less definite upper bound $w^{\prime} \lesssim 0.2 w(1+w)$ since a red shift $z \sim 1$ but evolving beyond $w^{\prime} \lesssim w(1+w)$ by the present. Again, $w \lesssim-0.8$ is a practical limit of applicability of these bounds.

We have analyzed the following tracker potentials for their freezing behavior: $V=M^{4+n} \phi^{-n}$ and $V=$ $M^{4+n} \phi^{-n} \exp \left(\alpha \phi^{2} / M_{P}^{2}\right)$ for $n>0$ [17, 18, 19, 20, 21]. The latter has an effective cosmological constant, but has been widely studied and so we consider it nonetheless, provided the field is closer to the origin than the non-zero minimum. Proposed tracker models such as $V=M^{4} \exp \left(M_{P} / \phi\right)$ do not have a zero minimum, and $V=M^{4}\left(\exp \left(M_{P} / \phi\right)-1\right)$ does not achieve $w \lesssim$ $-0.8, \Omega_{d e} \lesssim 0.8$. Other functions have been proposed as tracking potentials, but lack a firm basis in particle theory.

These distinct, physically motivated "thawing" and "freezing" behaviors are illustrated in Figure 1] while several examples of specific models are presented in Figure2 It would be quite useful to determine if one of these classes of physics phenomena is responsible for the dark energy accelerating our universe. We see that to distinguish thawing from freezing, a measurement resolution of order $\sigma\left(w^{\prime}\right) \approx(1+w)$ is required.

The question of the absolute level of deviation of $w$ from -1, i.e. the distinction from Einstein's cosmological constant, is less tractable. Certainly, one can obtain a scalar field solution, however unrealistic, at any given point in the $w-w^{\prime}$ phase space. Even for the thawing and freezing models, parameters may be finely tuned to keep $1+w$ arbitrarily close to zero within the shaded regions of Figure 1.

If the scalar field is prohibited from attaining values exceeding the Planck scale, lest quantum gravitational effects dominate, then there is a lower bound on $1+w$. Defining a characteristic scale $E \equiv|V /(d V / d \phi)|$ we demand $E<M_{P}$. Next, for a field rolling down its potential, we can express the scalar field equation of motion as

$$
w^{\prime}=-3\left(1-w^{2}\right)+(1-w) \frac{M_{P}}{E} \sqrt{\frac{3}{8 \pi} \Omega_{d e}(1+w)} .
$$

Taking $\Omega_{d e} \approx 0.7$ then thawing models must satisfy $1+$ $w \gtrsim 0.004$ whereas for freezing models $1+w \gtrsim 0.01$; this may be the limit of quintessence. These margins correspond to a $\sim 0.2 \%$ difference from a $\Lambda$ cosmology in distance to redshift $z=1$. Such an absolute precision goal is clearly extraordinarily challenging.

Note that early universe inflation can similarly approach pure exponential expansion, with its deviations broadly characterized by dynamics into models tilted to prefer large-scale or small-scale power, and important implications in the distinction 22. The structure we find in the canonical scalar-field phase-plane based on simple physical considerations may prove useful, and we have 
here presented firm targets for a basic test of dark energy. The language is different from inflation for two reasons: the dark energy need not be rolling as slowly as the inflaton, and the dark energy is not totally dominant, unlike the inflaton.

Charting the late-time cosmic evolution - through Type Ia supernovae distances, weak gravitational lensing probes of large-scale structure evolution, distance ratios from baryon acoustic oscillations in galaxy clustering, etc - is the subject of intense investigations. As a gauge of the requisite resolution, a $1 \%$ variation in luminosity distance to redshift $z=1$ distinguishes between: $\Lambda$ and $w=-0.95, w^{\prime}=0$; models which evolve along the top and bottom of the thawing region out to $w=-0.8$; models which evolve along the top and bottom of the freezing region in to $w=-0.95$. The goal of making the fun-

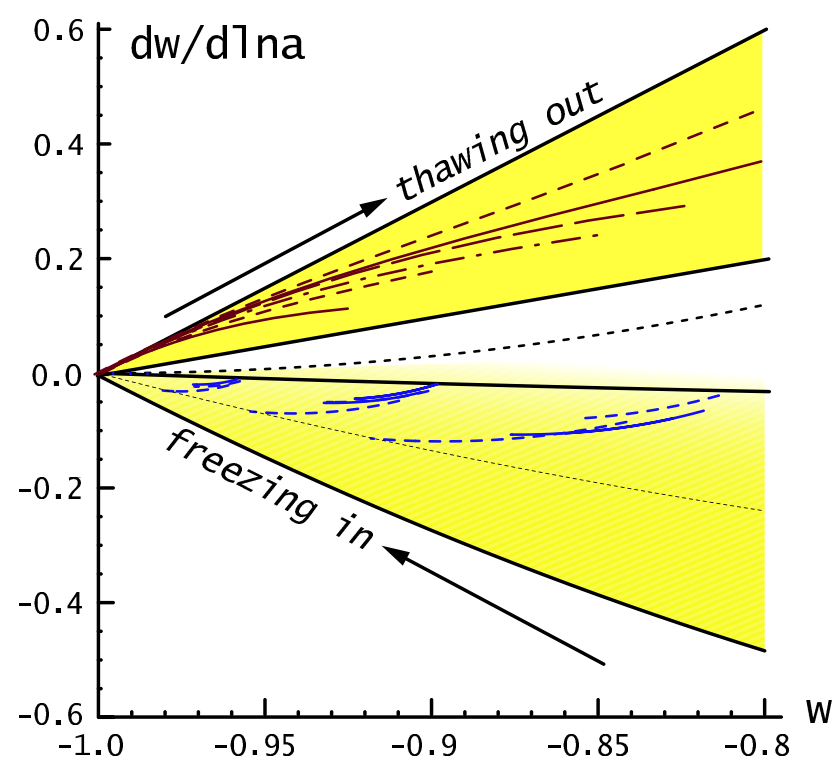

FIG. 2: The evolutionary tracks in $w-w^{\prime}$ phase space are shown for a variety of particle physics models of scalar fields. The two broad classes are clear: those that initially are frozen and look like a cosmological constant, starting at $w=-1$, $w^{\prime}=0$, and then thaw and roll to $w^{\prime}>0$, and those that initially roll and then slow to a creep as they come to dominate the Universe. The sample of thawing models shown have potentials $V \propto \phi^{n}$ for $n=1,2,4$ (short-, dot-, and long-dashed curves) and a PNGB with $V \propto \cos ^{2}(\phi / 2 f)$ (solid curves). The right-most point of the tracks corresponds to the present. For variety, the $n=4$ model has $\Omega_{d e}=0.6$, and the $n=1$ model ending at $w=-0.8$ has $\Omega_{d e}=0.65$. All other models end with a fractional energy density $\Omega_{d e}=0.7$. The sample of freezing models shown have potentials $V \propto \phi^{-n}, \phi^{-n} \mathrm{e}^{\alpha \phi^{2}}$ (solid and dashed curves). The line $w^{\prime}=1.5 w(1+w)$ indicated by the light, dotted line is a possible lower bound on the freezing models. The left-most point of the tracks corresponds to the present; the right-most point is at $z=1$. For variety, upper and lower close pairs of curves have $\Omega_{d e}=0.7,0.8$ respectively. All other models end with a fractional energy density $\Omega_{d e}=0.7$. damental physics distinction between the thawing and freezing regions is challenging but achievable in the next generation of experiments $23,24,25,26]$ if the dynamics is sufficiently apparent. In the case $1+w \gtrsim 0.05$, dedicated dark energy experiments now being designed, such as the Joint Dark Energy Mission, will probe cosmology with sufficient accuracy to be able to decide the issue.

This will probably not be the final word on dark energy ( $c f$. 27] ), but if the answer is not consistent with a cosmological constant then the rewards are obvious in discovering new physics beyond our current standard models. It is interesting to note that the fate of the Universe is very different for the case of a thawing field, as the acceleration is temporary, as compared to a freezing field, for which the acceleration continues unabated. If the result lies outside the two phase-space regions categorized here then we may have to look beyond simple explanations, perhaps to even more exotic physics such as a modification of Einstein gravity.

This work is supported by NSF AST-0349213 at Dartmouth, and by DOE AC03-76SF00098 at LBL. EL thanks Dartmouth for its hospitality.

[1] R. R. Caldwell, Phys. World 17, 37 (2004).

[2] T. Padmanabhan, Phys. Rept. 380, 235 (2003).

[3] P.J.E. Peebles, B. Ratra, Rev. Mod. Phys. 75, 559 (2003).

[4] B. Ratra and P. J. Peebles, Phys. Rev. D 37, 3406 (1988).

[5] C. Wetterich, Nucl. Phys. B 302, 668 (1988).

[6] J. A. Frieman, C. T. Hill, A. Stebbins and I. Waga, Phys. Rev. Lett. 75, 2077 (1995).

[7] K. Coble, S. Dodelson and J. A. Frieman, Phys. Rev. D 55, 1851 (1997).

[8] R. R. Caldwell, R. Dave and P. J. Steinhardt, Phys. Rev. Lett. 80, 1582 (1998).

[9] R. A. Knop et al. [The Supernova Cosmology Project Collaboration], Astrophys. J. 598, 102 (2003).

[10] A. G. Riess et al. [Supernova Search Team Collaboration], Astrophys. J. 607, 665 (2004).

[11] U. Seljak et al., arXiv:astro-ph/0407372

[12] R. Kallosh, J. Kratochvil, A. Linde, E.V. Linder and M. Shmakova, JCAP 0310, 015 (2003).

[13] T. Barreiro, E. J. Copeland and N. J. Nunes, Phys. Rev. D 61, 127301 (2000).

[14] P. G. Ferreira and M. Joyce, Phys. Rev. D 58, 023503 (1998).

[15] I. Zlatev, L. M. Wang and P. J. Steinhardt, Phys. Rev. Lett. 82, 896 (1999).

[16] P.J. Steinhardt, "Proceedings of the the Pritzker Symposium on Status of Inflationary Cosmology," ed. by M. Turner, (U. Chicago Press, Chicago, 2000).

[17] P. Binetruy, Phys. Rev. D 60, 063502 (1999).

[18] P. Brax and J. Martin, Phys. Lett. B 468, 40 (1999).

[19] A. Masiero, M. Pietroni and F. Rosati, Phys. Rev. D 61, 023504 (2000).

[20] E. J. Copeland, N. J. Nunes and F. Rosati, Phys. Rev. D 62, 123503 (2000). 
[21] A. de la Macorra and C. Stephan-Otto, Phys. Rev. Lett. 87, 271301 (2001).

[22] W.H. Kinney, E.W. Kolb, A. Melchiorri, and A. Riotto, Phys. Rev. D 69, 103516 (2004).

[23] G. Aldering et al., PASP submitted, arXiv:astro-ph/0405232

[24] M. Jarvis, B. Jain, G. Bernstein and D. Dolney,
arXiv:astro-ph/0502243

[25] E. V. Linder and R. Miquel, Phys. Rev. D 70, 123516 (2004).

[26] A. Upadhye, M. Ishak and P. J. Steinhardt, arXiv:astro-ph/0411803

[27] I. Maor and R. Brustein, Phys. Rev. D 67, 103508 (2003). 